\title{
MicroRNA-26a inhibits cell proliferation and invasion by targeting FAM98A in breast cancer
}

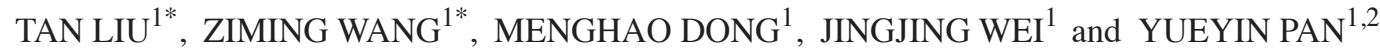 \\ ${ }^{1}$ Department of Oncology, Provincial Hospital Affiliated to Anhui Medical University, Hefei, Anhui 230032; \\ ${ }^{2}$ Department of Oncology, The First Affiliated Hospital of The University of Science and Technology of China, \\ Division of Life Sciences and Medicine, University of Science and Technology of China, Hefei, Anhui 230001, P.R. China
}

Received October 14, 2020; Accepted February 18, 2021

DOI: $10.3892 / \mathrm{ol} .2021 .12628$

\begin{abstract}
MicroRNAs (miRNAs/miRs) play key roles in cancer progression. Extensive research has revealed that miR-26a is abnormally expressed and functions as a tumor suppressor in numerous types of cancer. Thus, the present study was undertaken to investigate the regulatory role and potential mechanism of action of miR-26a in breast cancer. Furthermore, the present study aimed to examine the alterations in miR-26a expression and its effects on human breast cancer cells. Reverse transcription-quantitative PCR was conducted to assess the differences in miR-26a expression between human breast cancer and normal breast specimens. A Cell Counting Kit-8 assay and cloning experiments were used to detect cell proliferation and clone formation. Wound healing and Transwell assays were performed to examine cell migration and invasion. A luciferase activity experiment was utilized to validate the association between miR-26a and family with sequence similarity 98 member A (FAM98A). Western blotting was conducted to detect the protein expression levels of FAM98A, sonic hedgehog signaling molecule (SHH), smoothened, frizzled class receptor (SMO) and GLI family zinc finger 1 (GLI1). The results indicated that miR-26a expression was decreased in breast carcinoma tissues and
\end{abstract}

Correspondence to: Professor Yueyin Pan, Department of Oncology, Provincial Hospital Affiliated to Anhui Medical University, 17 Lujiang Road, Hefei, Anhui 230032, P.R. China

E-mail: panyueyin@ustc.edu.cn

*Contributed equally

Abbreviations: miRNAs/miR, microRNAs; PRMT, protein arginine methyltransferases; NC, negative control; CCK-8, Cell Counting Kit-8; 3'-UTR, 3'-untranslated region; RT-qPCR, reverse transcription-quantitative PCR; FAM98A, family with sequence similarity 98 member A; SHH, sonic hedgehog signaling molecule; SMO, smoothened, frizzled class receptor; GLI1, GLI family zinc finger 1

Key words: breast cancer, miR-26a, proliferation, invasion, FAM98A cell lines. Moreover, overexpression of miR-26a significantly suppressed cell proliferation, clone formation ability and metastasis, and it sensitized breast cancer cells to docetaxel. It was demonstrated that miR-26a directly targeted FAM98A, and that FAM98A, SHH, SMO and GLI1 expression levels were decreased in cells transfected with miR-26a mimics. Collectively, the results of the present study suggested that miR-26a negatively regulated the expression of FAM98A, indicating that it may play a key role in the suppression of breast carcinogenesis.

\section{Introduction}

Breast cancer is an intractable type of cancer that poses a major threat to the physical and mental wellbeing of women (1), and its incidence rate is increasing annually worldwide. Advanced diagnostic methods and comprehensive treatment contribute to effectively improving the prognosis of patients with early-stage breast cancer (2). Moreover, a significant proportion of patients are treated with docetaxel chemotherapy. Docetaxel, a cytotoxic drug that belongs to the taxane family of anticancer agents, is employed for the treatment of various cancers, including breast cancer, and acts via interfering in tubulin synthesis (3). Patients with advanced breast cancer receiving long- term chemotherapy may develop resistance to the initial anticancer drugs, which results in treatment failure, tumor recurrence and metastasis, and increased mortality risk $(4,5)$. Therefore, an improved understanding of the regulatory mechanisms underlying breast cancer progression and the identification of novel effective therapeutic strategies are urgently required to improve the prognosis.

MicroRNAs (miRNAs/miRs) are a class of RNAs in eukaryotes that are 18-25 nt in length, lack protein-coding ability, and have been attracting increasing interest in the field of cancer research. miRNAs are involved in the post-transcriptional regulation of numerous human genes via negative regulation of target gene expression, promotion of target mRNA cleavage and suppression of translation of proteins that may serve as direct regulators in biological processes (6). Previous studies have reported a strong association between miRNAs and numerous biological processes, such as inflammation, stress response, cell cycle regulation, cell proliferation, differentiation, invasion and drug resistance (7-11). miR-26a 
has been reported to act as a cancer promoter in some carcinomas, and to suppress tumor occurrence and development in other types of cancer (12). A recent study revealed that miR-26a expression is downregulated in breast cancer and is considered to act as a tumor suppressor (13). However, the underlying mechanisms through which miRNAs regulate the cancer development process require further investigation.

Several studies have reported that the abnormal expression of family with sequence similarity 98 member A (FAM98A) is associated with multiple types of cancer, including ovarian, endometrial, colorectal and lung cancer (14-17). Akter et al (14) revealed that FAM98A, which is arginine-methylated by protein arginine methyltransferase 1 (PRMT1), was highly expressed in ovarian cancer cell lines, and found that the knockdown of FAM98A reduced ovarian cancer cell migration, invasion and colony formation. Another study conducted by Akter et al demonstrated that FAM98A and FAM98B, a new complex binding to DDX1 and C14orf166, are required for PRMT1 expression. FAM98A and PRMT1, which are highly expressed in colorectal cancer tissues, act as tumor promoters and facilitate cancer occurrence and progression (15). A recent study revealed that FAM98A promoted cancer cell survival and progression of endometrial carcinoma, whereas its overexpression was associated with poor prognosis (16). Moreover, another study identified an association between the presence of FAM98A and advanced lung cancer. FAM98A was shown to act as an oncogene by activating the P38/activating transcription factor 2 signaling pathway to promote lung cancer cell proliferation and colony formation (17).

The present study was undertaken to investigate the role of miR-26a in breast cancer by examining its expression and detecting its effect on cell proliferation, colony formation, migration and invasion. Furthermore, the association between miR-26a and FAM98A was elucidated using bioinformatics analysis, luciferase reporter assay and western blotting.

\section{Materials and methods}

Patients and specimens. A total of 13 pairs of breast cancer and matched non-cancerous breast tissues were obtained from female patients, aged 30-73 years, undergoing surgery for breast cancer at The First Affiliated Hospital of University of Science and Technology of China (Hefei, China) between September 2019 and August 2020. Corresponding non-cancerous breast tissues at a distance of $>5 \mathrm{~cm}$ from the edge of the tumor were collected. These tissue specimens were freshly frozen in liquid nitrogen and stored at $-80^{\circ} \mathrm{C}$. Written informed consent was obtained from the patients, and the study was approved by the Ethics Committee of The First Affiliated Hospital of University of Science and Technology of China (approval no. 2019-ky086). The clinicopathological characteristics of the patients are summarized in Table I.

Cell lines and cell culture. The human breast cancer cell lines SK-BR-3, BT474, MDA-MB-231, MDA-MB-468 and MCF-7, and the non-tumorigenic epithelial cell line MCF-10A were obtained from the American Type Culture Collection. Cells were grown in RPMI-1640 medium (Gibco; Thermo Fisher Scientific, Inc.) supplemented with 10\% FBS (Gibco; Thermo Fisher Scientific, Inc.) and $100 \mathrm{U} / \mathrm{ml}$ penicillin and streptomycin (Gibco; Thermo Fisher Scientific, Inc.) in a humidified incubator at $37^{\circ} \mathrm{C}$ with $5 \% \mathrm{CO}_{2}$.

Cell transfection. Briefly, 1 day prior to transfection, MCF-7 and MDA-MB-231 cells $\left(1 \times 10^{5}\right.$ cells/well) were seeded into 6-well plates with complete growth medium and no antibiotics. The cells were then transfected with miR-26a mimic and negative control (NC) (Shanghai GenePharma Co., Ltd.) for $24 \mathrm{~h}$ at room temperature using Lipofectamine ${ }^{\circledR} 2000$ (Invitrogen; Thermo Fisher Scientific, Inc.) according to the manufacturer's protocol. The concentration used for miR-26a mimic was $100 \mathrm{nM}$. The sequences of the miR-26a mimics and NC used were as follows: miR-26a: 5'-UUCAAGUAAUCCAGGAUA GGCU-3'/5'-CCUAUCCUGGAUUACUUGAAUU-3' and NC: 5'-UUCUCCGAACGUGUCACGUTT-3'/5'-ACGUGA CACGUUCGGAGAATT-3'. RT-qPCR was conducted to verify whether the transfection of miR-26a mimic significantly increased miR-26a expression in MCF-7 and MDA-MB-231 cells. Subsequent experiments were carried out 24-72 h after transfection.

Total RNA extraction and reverse transcription-quantitative $P C R(R T-q P C R)$ analysis. Total RNA was extracted from the tissue samples and cell lines using TRIzol ${ }^{\circledR}$ reagent (Invitrogen; Thermo Fisher Scientific, Inc.) and was quantified using an ultraviolet spectrophotometer (NanoDrop 2000; Thermo Fisher Scientific, Inc.). cDNA was synthesized using a Reverse Transcription kit (Shanghai GenePharma Co., Ltd.) according to the manufacturer's protocol. All PCR reagents and primers were designed and synthesized by Shanghai GenePharma Co., Ltd. cDNAs were used as templates in a $20-\mu 1$ PCR reaction using a LightCycler480II system (Roche Diagnostics). The reaction mixture was as follows: cDNA $(2 \mu \mathrm{l})$, miRNA/U6 snRNA specific primer set $(0.4 \mu \mathrm{l}), 5 \mathrm{U} / \mu \mathrm{l}$ Taq DNA polymerase $(0.2 \mu \mathrm{l}), 2 \mathrm{X}$ Real-time PCR Master Mix (containing SYBR Green) $(10 \mu \mathrm{l})$ and sterilized $\mathrm{H}_{2} \mathrm{O}(7.4 \mu \mathrm{l})$. U6 was used as an internal reference. The primers used were as follows: miR-26a forward, 5'-CTCCTCGCTTCAAGTAATCCAG-3', and reverse, 5'-TATGCTTGTTCTCGTCTCTGTGTC-3'; and U6 forward, 5'-CAGCACATATACTAAAATTGGAAC G-3' and reverse, 5'-ACGAATTTGCGTGTCATCC-3'. The thermocycling conditions were as follows: Initial denaturation at $95^{\circ} \mathrm{C}$ for $3 \mathrm{~min}, 40$ cycles at $95^{\circ} \mathrm{C}$ for $12 \mathrm{sec}$ and $62^{\circ} \mathrm{C}$ for $40 \mathrm{sec}$. The results were quantified using the $2^{-\Delta \Delta \mathrm{Cq}}$ method (18).

Western blot analysis. Total protein was extracted from MCF-7 and MDA-MB-231 cells transfected with miR-26a mimic and miR-NC, respectively using RIPA lysis buffer (Beyotime Institute of Biotechnology Co., Ltd.) and the concentration of total protein was determined with the BCA protein assay kit (Pierce; Thermo Fisher Scientific, Inc.). Protein samples $(25 \mu \mathrm{g})$ were separated via 10\% SDS-PAGE and blotted onto PVDF membranes (EMD Millipore). The membranes were blocked with 5\% non-fat milk for $1 \mathrm{~h}$ at room temperature and incubated with primary antibodies against FAM98A (dilution 1:500; ab204083), sonic hedgehog (Hh) signaling molecule (SHH; dilution 1:1,000; ab53281), smoothened, frizzled class receptor (SMO; dilution 1:1,000; ab124964), GLI family zinc finger 1 (GLI1; dilution 1:1,000; ab134906) and GAPDH (dilution 1:2,000; ab8245) (all from Abcam) overnight at $4^{\circ} \mathrm{C}$. 
Table I. Clinicopathological characteristics of patients with breast cancer $(n=13)$.

\begin{tabular}{|c|c|}
\hline Characteristics & No. of patients \\
\hline \multicolumn{2}{|l|}{ Age (years) } \\
\hline$\leq 50$ & 7 \\
\hline$>50$ & 6 \\
\hline \multicolumn{2}{|l|}{ Tumor size (cm) } \\
\hline$\leq 2$ & 5 \\
\hline$>2$ & 8 \\
\hline \multicolumn{2}{|c|}{ Lymph node status } \\
\hline Negative & 5 \\
\hline Positive & 8 \\
\hline \multicolumn{2}{|c|}{ Pathological stage } \\
\hline I-II & 8 \\
\hline III-IV & 5 \\
\hline \multicolumn{2}{|c|}{ Estrogen receptor status } \\
\hline Negative & 6 \\
\hline Positive & 7 \\
\hline \multicolumn{2}{|c|}{ Progesterone receptor status } \\
\hline Negative & 8 \\
\hline Positive & 5 \\
\hline \multicolumn{2}{|c|}{$\begin{array}{l}\text { Human epidermal growth factor } \\
\text { receptor- } 2 \text { status }\end{array}$} \\
\hline Negative & 8 \\
\hline Positive & 5 \\
\hline \multicolumn{2}{|l|}{ Ki-67 } \\
\hline$\leq 15 \%$ & 0 \\
\hline$>15 \%$ & 13 \\
\hline
\end{tabular}

The membranes were subsequently incubated with horseradish peroxidase (HRP)-conjugated secondary antibody (dilution 1:2,000; cat. nos. 58802 and 93702; Cell Signaling Technology, Inc.) at room temperature for $1 \mathrm{~h}$. The western blots were visualized with enhanced chemiluminescence reagents (EMD Millipore).

Tumor sphere formation assay. At $24 \mathrm{~h}$ post-transfection, MCF-7 and MDA-MB-231 cells were seeded in 6-well plates at a density of 2,000 cells/well with ultra-low attachment surface polystyrene (Corning, Inc.). Cells were cultured in DMEM-F12 (BioWhittaker), supplemented with B27 (1:50, Invitrogen; Thermo Fisher Scientific, Inc.), $20 \mathrm{ng} / \mathrm{m}$ basic fibroblast growth factor and $20 \mathrm{ng} / \mathrm{ml}$ epidermal growth factor (BD Biosciences). After incubation for 4 and 9 days in a 5\% $\mathrm{CO}_{2}$ incubator, the number of tumor spheres was counted and images were captured using a light microscope (Olympus Corporation; magnification, x100).

Cell proliferation assay. A Cell Counting Kit-8 (CCK-8; Dojindo Molecular Technologies, Inc.) assay was conducted for cell viability and drug toxicity analysis according to the manufacturer's protocol., MCF-7 and MDA-MB-231 cells were seeded into 96-well microplates at a density of 3,000 cells per well with or without different concentrations $(0.1,0.5,1.0$ or $1.5 \mu \mathrm{M}$ ) of docetaxel $24 \mathrm{~h}$ after transfection. After the cells had been incubated at $37^{\circ} \mathrm{C}$ for $24,48,72$ and $96 \mathrm{~h}, 10 \mu \mathrm{lCCK}-8$ solution was added into each well and incubated at $37^{\circ} \mathrm{C}$ for $2 \mathrm{~h}$ in an incubator. Cell proliferation was detected on an INFINITE 200 Promultimode reader (Tecan Group, Ltd.) with the optical density measured at $450 \mathrm{~nm}$.

Colony formation assay. At $24 \mathrm{~h}$ post-transfection, cells in the logarithmic growth phase were collected and seeded in 6-well plates (500 cells per well). After incubation for 2 weeks at $37^{\circ} \mathrm{C}$ in a $5 \% \mathrm{CO}_{2}$ incubator, the medium was discarded. The cells were fixed in $4 \%$ paraformaldehyde for $15 \mathrm{~min}$ at room temperature after being rinsed twice with PBS, and were then stained with $0.1 \%$ crystal violet solution for $30 \mathrm{~min}$ at room temperature. The number of cells per clone ( $>50$ cells) was counted under a light microscope (Olympus Corporation; magnification, $\mathrm{x} 40$ ).

Cell migration and invasion assay. MCF-7 and MDAMB-231 cells were seeded in a 6-well plate at a density of $4 \times 10^{5}$ cells/well and were cultured overnight. After transfection, when the cell confluence reached $\sim 90 \%$, a $200-\mu 1$ pipette was used to scratch the wells. Then, the cells were rinsed twice with PBS and cultured with serum-free RPMI-1640 medium (Gibco; Thermo Fisher Scientific, Inc.). At 0 and $48 \mathrm{~h}$ after scratching, the wound healing was imaged using an inverted fluorescence microscope (Olympus Corporation; magnification, $\mathrm{x} 10$ ).

The Transwell assay was conducted using Transwell inserts in 24-well plates (pore size, $8 \mu \mathrm{m}$; Corning, Inc.). The upper surface of the membrane was pre-coated with Matrigel (BD Biosciences) at $37^{\circ} \mathrm{C}$ for $30 \mathrm{~min}$. A total of $1 \times 10^{5}$ cells suspended in RPMI-1640 medium without FBS were seeded into the upper chamber, and the lower chamber was filled with cell medium supplemented with $10 \%$ FBS. After incubation for $48 \mathrm{~h}$, the cells invading in the lower chamber were fixed in $4 \%$ paraformaldehyde for $15 \mathrm{~min}$ at room temperature and stained with $0.1 \%$ crystal violet solution for $30 \mathrm{~min}$ at room temperature. Then, invading cells were counted and imaged using an inverted fluorescence microscope (Olympus Corporation; magnification, x100).

Luciferase reporter assay. The target binding sites of miR-26a in FAM98A 3'-UTR were predicted using TargetScanHuman 7.2 (http://www.targetscan.org). The FAM98A 3'-UTR containing miR-26a sequences binding sites was amplified and cloned into the pGL3 Basic vector (Promega Corporation). Then, pGL3-FAM98A 3'-UTR-wild-type (WT) and pGL3-FAM98A 3'-UTR-mutant (MT) (100 ng) were co-transfected with miR-NC or miR-26a mimic (100 nM) into MCF-7 and MDA-MB-231 cells using Lipofectamine ${ }^{\circledR} 2000$ according to the manufacturer's protocol. After transfection for 48 h, a Dual-Luciferase Reporter Assay system (Promega Corporation) was used to detect luciferase activity. Renilla luciferase activity was used for normalization.

Statistical analysis. Data management and analysis were performed using SPSS 23.0 (IBM, Corp.). P<0.05 was considered to indicate a statistically significant difference. 
A

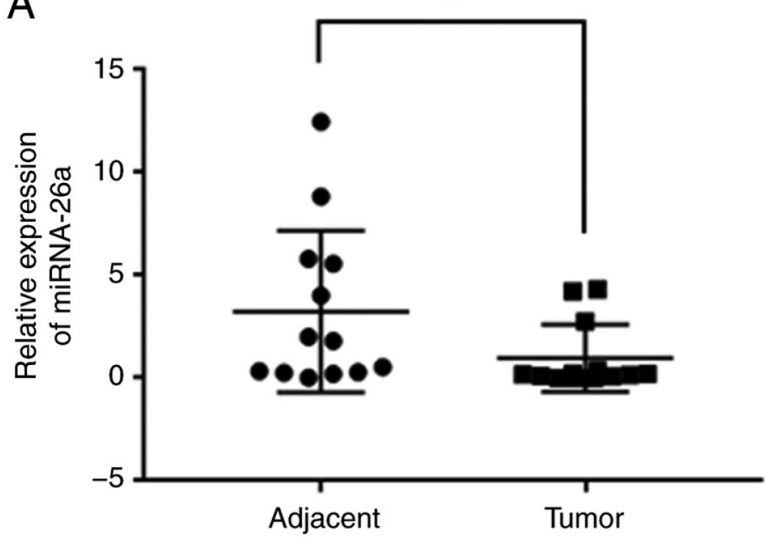

C

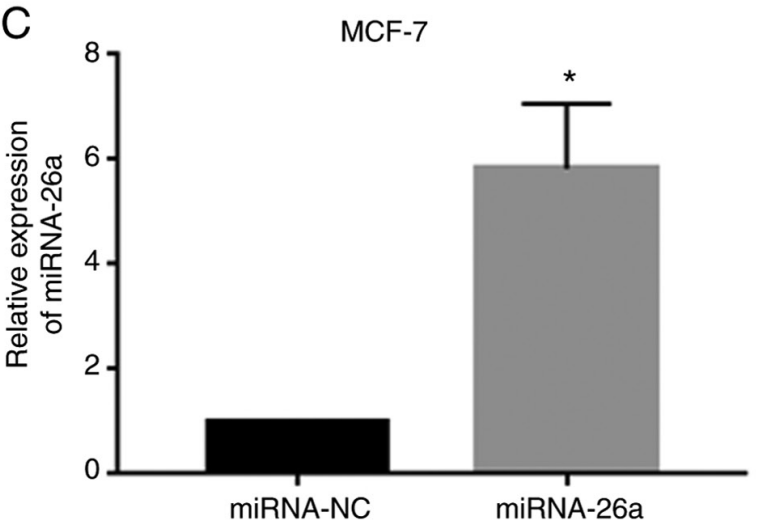

B
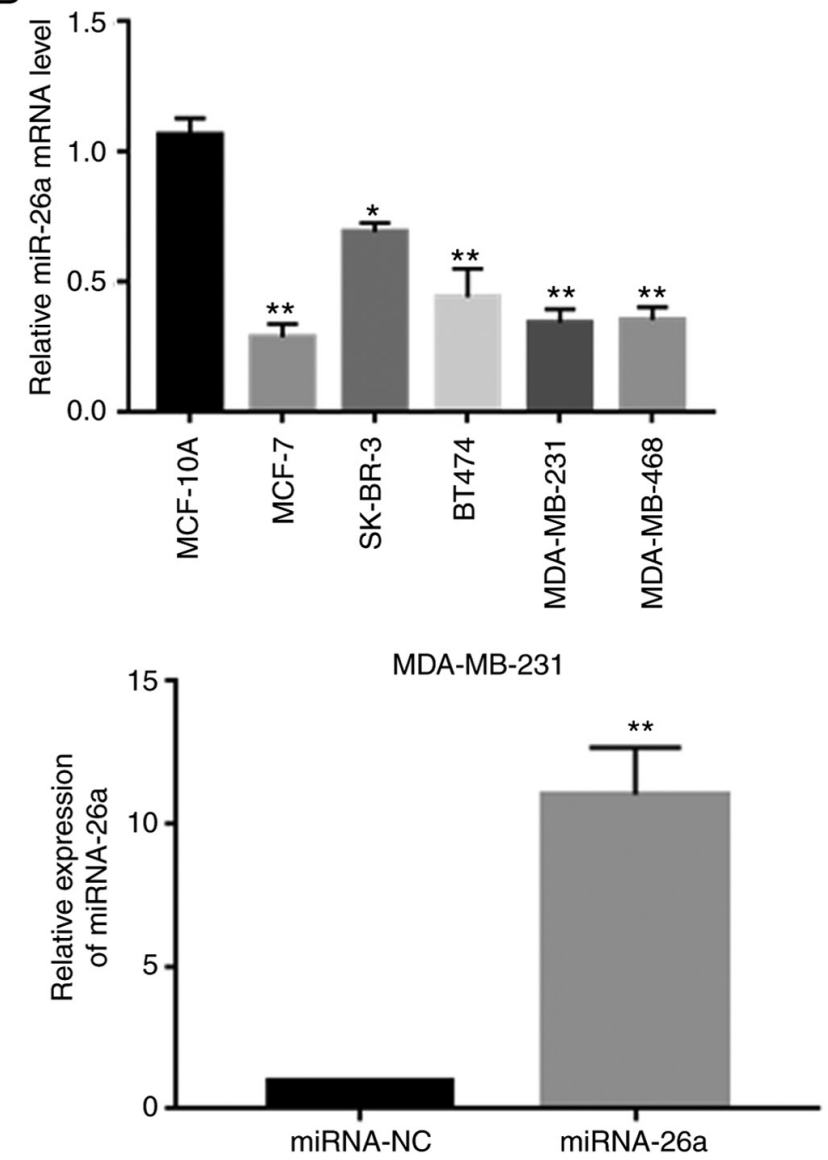

Figure 1. miR-26a is downregulated in human breast cancer tissues and cell lines. Transfection of miR-26a mimic significantly increased miR-26a expression. (A) miR-26a expression in breast cancer specimens and adjacent non-tumor breast tissues was examined via RT-qPCR. "P<0.05 vs. matched non-tumor breast tissues. (B) miR-26a expression in breast cancer cell lines and a non-tumorigenic epithelial cell line was analyzed using RT-qPCR. ${ }^{*} \mathrm{P}<0.05$, ${ }^{* *} \mathrm{P}<0.01$ vs. MCF-10A. (C) MCF-7 and MDA-MB-231 cells were transfected with miR-26a mimic and miR-NC and the relative expression of miR-26a was detected 72 h later. ${ }^{*} \mathrm{P}<0.05,{ }^{* *} \mathrm{P}<0.01$ vs. NC. miR, microRNA; RT-qPCR, reverse transcription-quantitative PCR; NC, negative control.

Two-tailed unpaired Student's t-test was used to analyze the association between two independent groups. Statistically significant differences among multiple groups were determined using one-way ANOVA followed by Tukey's post hoc test. To assess differences in cell proliferation curves, repeated-measures ANOVAs was used. All cell biological assays were evaluated in triplicate.

\section{Results}

miR-26a is downregulated in human breast cancer tissues and cell lines. RT-qPCR analysis was conducted to determine the differential expression of miR-26a in 13 pairs of clinical tissue specimens. The results indicated that the relative mRNA expression level of miR-26a was decreased in cancer tissues compared with that in the adjacent non-cancerous tissues (Fig. 1A). Moreover, further experiments demonstrated that miR-26a expression was upregulated in MCF-10A cells and was downregulated in all breast carcinoma cell lines (MDA-MB-468, MDA-MB-231, BT474, SK-BR-3 and MCF-7), particularly in MCF-7 and MDA-MB-231 cells (Fig. 1B). The results indicated that the expression level of miR-26a may be associated with breast carcinogenesis and metastasis.
Overexpression of miR-26a inhibits cell proliferation and colony formation in breast cancer and enhances the sensitivity of cancer cells to docetaxel. In the present study, it was observed that miR-26a expression was significantly decreased in the MCF-7 and MDA-MB-231 cell lines (Fig. 1B). Therefore, these two cell lines were used to examine the role of miR-26a in breast cancer cells. Subsequently, miR-26a mimic and miR-NC were transfected into the breast cancer cells to determine whether miR-26a affected cell proliferation and colony formation. The transfection of miR-26a mimic significantly increased miR-26a expression compared with that observed in the miR-NC group (Fig. 1C). Proliferation assay results suggested that the proliferation of cells transfected with miR-26a mimic was decreased (Fig. 2A) and the overexpression of miR-26a sensitized carcinoma cells to docetaxel (Fig. 2B). Furthermore, sphere formation assay was performed to evaluate weather miR-26a modified the ability of the cell lines to grow in suspension as tumor spheres. However, there was no significant difference compared with the control group (data not shown). Moreover, the effect of miR-26a on the colony formation ability of cancer cells was examined, and it was found that the number of the colonies in the miR-26a mimic group was lower compared with that in the miR-NC group (Fig. 3A). 
A

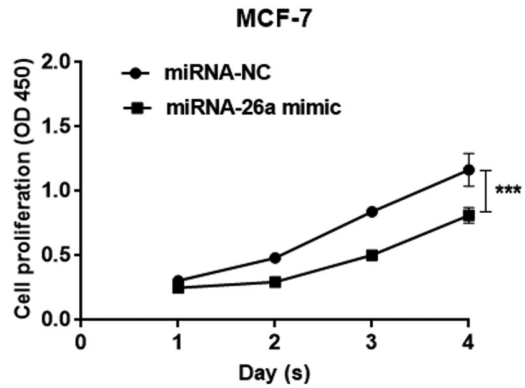

B

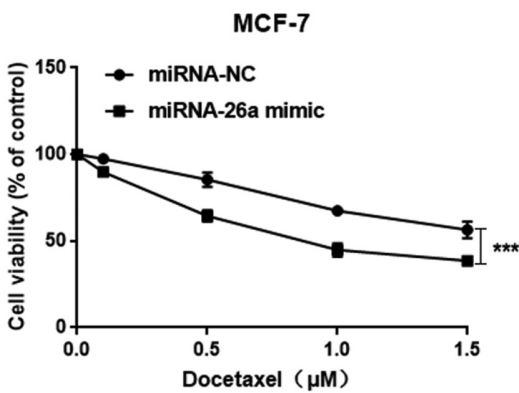

MDA-MB-231

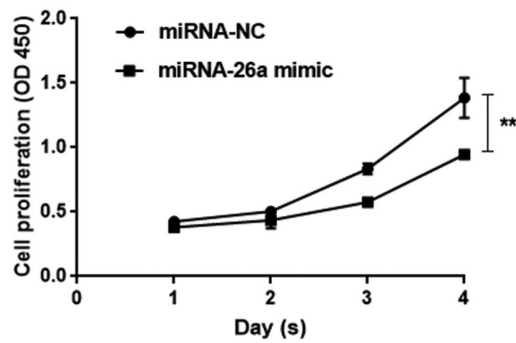

MDA-MB-231

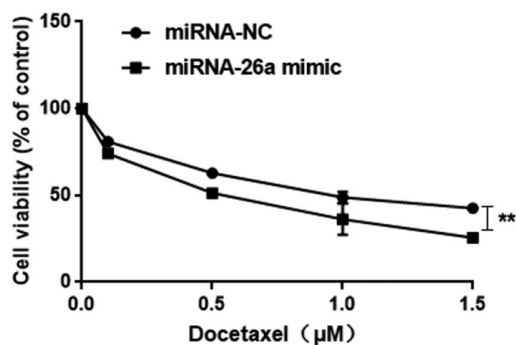

Figure 2. Overexpression of miR-26a inhibits the proliferation and enhances the sensitivity of breast cancer cells to docetaxel. (A) The proliferative ability in MCF-7 and MDA-MB-231 cells was detected using Cell Counting Kit-8 assays after the cells were transfected with miR-26a mimic and miR-NC. (B) The viability of MCF-7 and MDA-MB-231 cells was analyzed after treatment with different concentrations of docetaxel for $48 \mathrm{~h} .{ }^{* *} \mathrm{P}<0.01,{ }^{* * * *} \mathrm{P}<0.001$ vs. NC. miR, microRNA; NC, negative control.

A

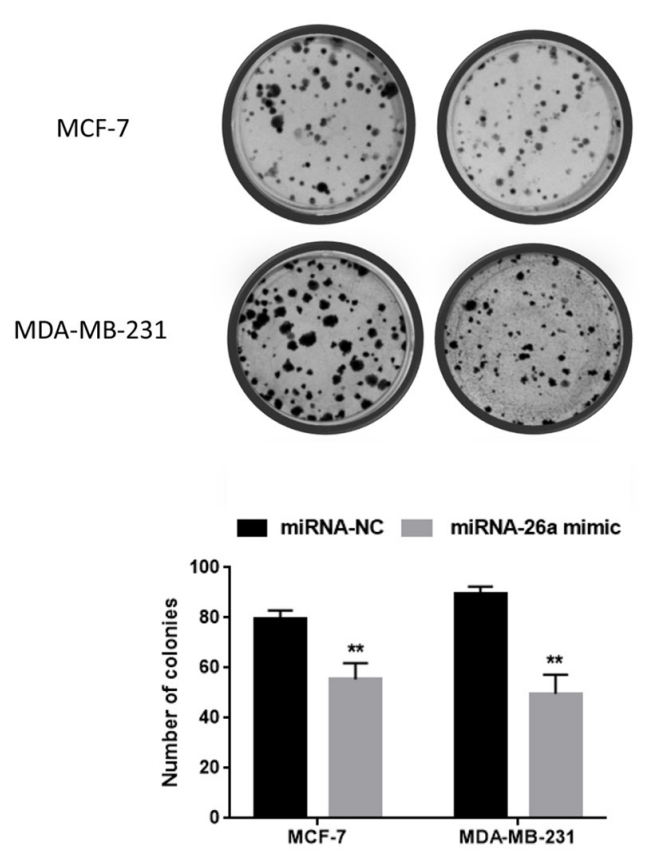

B

MCF-7
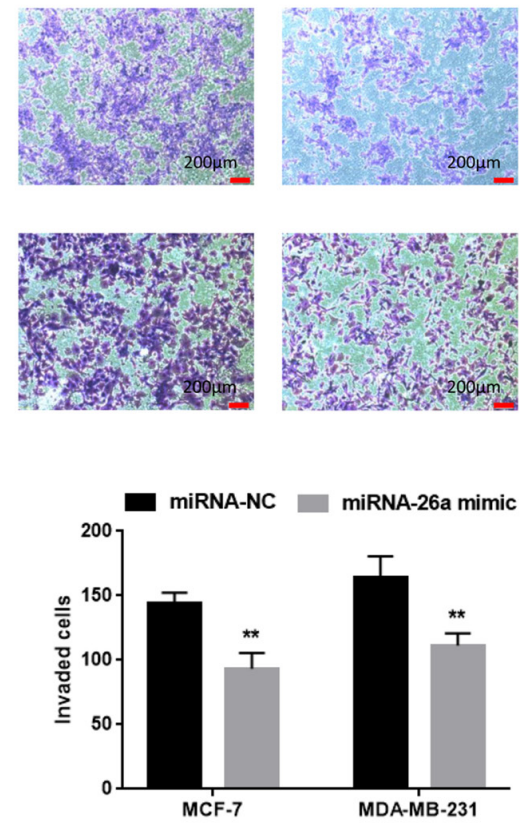

Figure 3. Overexpression of miR-26a suppresses cell colony formation and invasion. (A) MCF-7 and MDA-MB-231 cells transfected with miR-26a mimic or miR-NC were subjected to a colony formation assay. (B) The effect of miR-26a on cell invasion in cells transfected with miR-26a mimic was examined $48 \mathrm{~h}$ after transfection. ${ }^{* *} \mathrm{P}<0.01$ vs. NC. miR, microRNA; NC, negative control. Scale bar, $200 \mu \mathrm{m}$.

Overexpression of miR-26a suppresses cell migration and invasion. To determine the effects of miR-26a on cell migration and invasion, a series of transfections were performed. Transwell assay was used to assess cell invasion. As shown in Fig. 3B, the number of invading cells among breast cancer cells transfected with miR-26a mimic was lower compared with that of control cells. Moreover, cell migration was examined, and the results suggested that the migration of miR-26a mimic-transfected cells was markedly suppressed compared with that of the NC group (Fig. 4A). Collectively, these results indicated that miR-26a may attenuate breast carcinogenesis and progression. 
A MCF-7

miR-NC
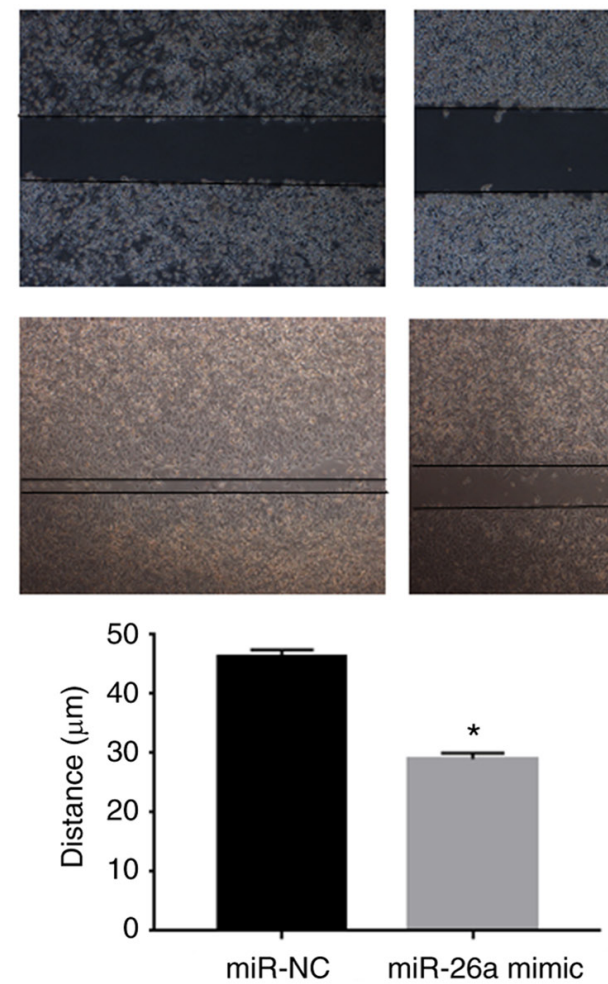

B miR-26a
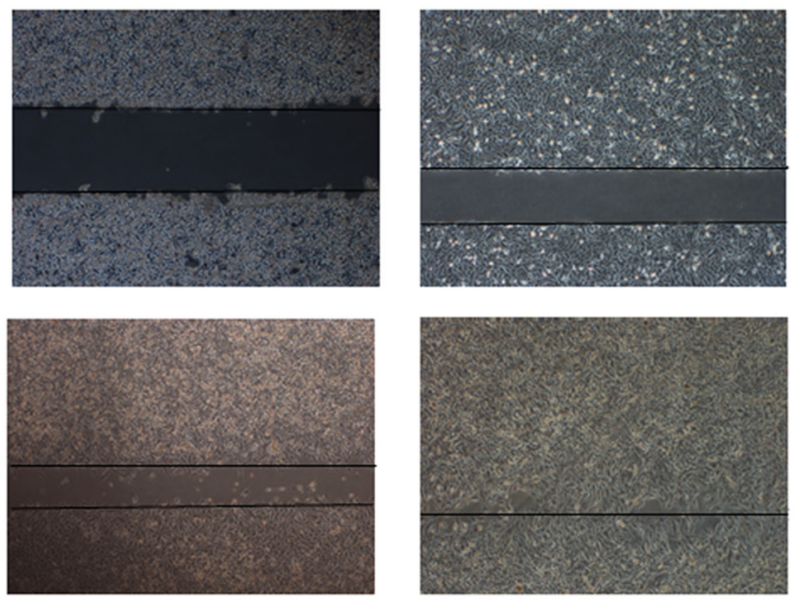

miR-NC

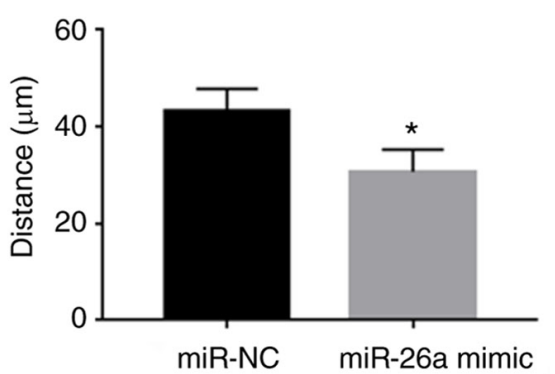

miR-26a
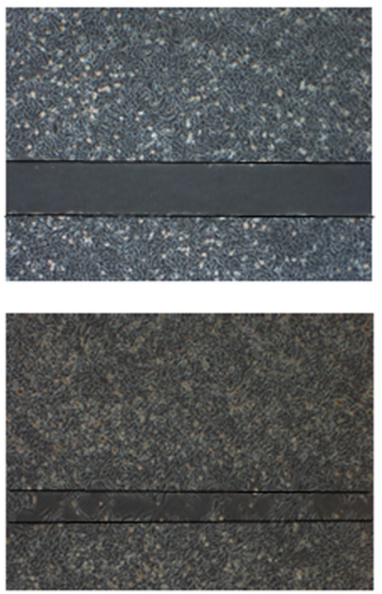

Figure 4. Transfection of miR-26a mimic inhibits cancer cell migration. (A and B) Confluent monolayers of miR-26a mimic-transfected MCF-7 and MDA-MB-231 cells were scratched, and the effect of miR-26a on cell migration was detected $48 \mathrm{~h}$ later. " $\mathrm{P}<0.05 \mathrm{vs.} \mathrm{NC.} \mathrm{miR,} \mathrm{microRNA;} \mathrm{NC,} \mathrm{negative} \mathrm{control.}$

A

\author{
Wild-type FAM98A \\ miR-26a
}

MUT FAM98A

C

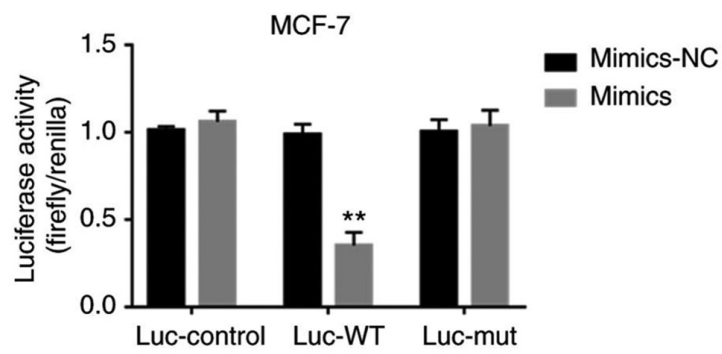

Position 94-101 of FAM98A 3'--UTR

5' ...AAUCGAACCUCCACCCCCAGAGA... 3'

|||||| $\mid$

$3^{\prime} \ldots$ GACCUCCUUCCCGGGUCUCC... 5'

|||||| $\mid$
B

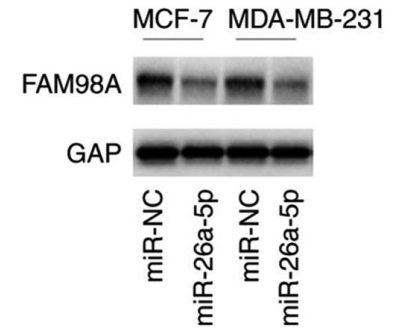

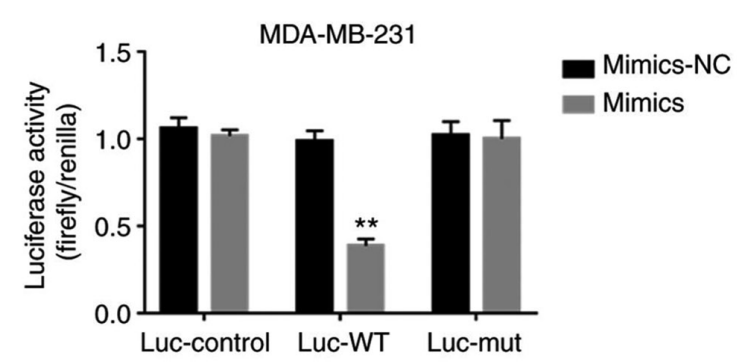

Figure 5. miR-26a directly targets FAM98A in breast cancer. (A) A TargetScan algorithm was used to predict the binding sites of miR-26a-5p in FAM98A 3'-UTR. (B) FAM98A protein expression was examined via western blotting in miR-26a-overexpressing cells. (C) Cells co-transfected with the WT 3'-UTR of FAM98A, the MT 3'-UTR of FAM98A and miR-26a were subjected to the luciferase activity assay. ${ }^{* *} \mathrm{P}<0.01 \mathrm{vs}$. NC. miR, microRNA; NC, negative control; UTR, untranslated region; WT, wild-type; MT, mutant; FAM98A, family with sequence similarity 98 member A.

miR-26a directly targets FAM98A in breast cancer. In order to understand how miR-26a regulates tumorigenesis and cancer progression after identifying the functional role of miR-26a, bioinformatics analysis (TargetScan algorithm) was utilized to identify the putative target of miR-26a. It was found that
miR-26a could directly target FAM98A (Fig. 5A). A luciferase reporter assay was then conducted to test whether miR-26a binds to the 3'-UTR of FAM98A. The results suggested that miR-26a significantly repressed the relative luciferase activity of the WT 3'-UTR of FAM98A, whereas no significant 
A

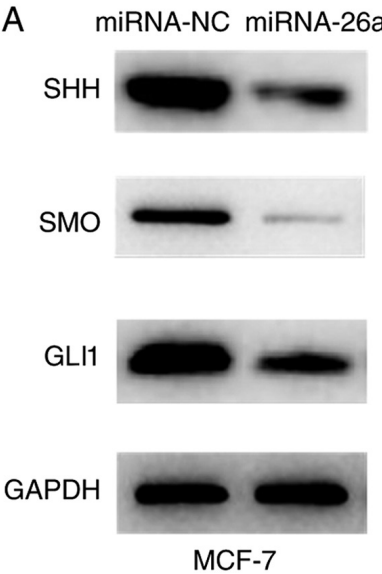

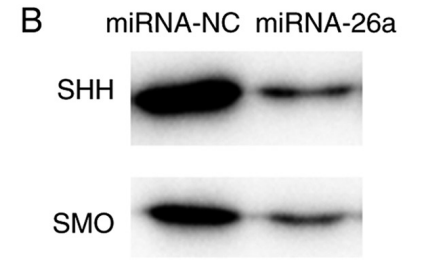

GLI1

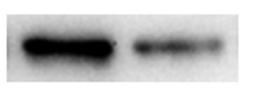

GAPDH

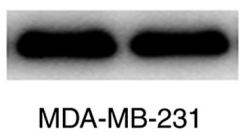

Figure 6. miR-26a suppresses the Hedgehog signaling pathway. SHH, SMO and GLI1 protein expression levels in (A) MCF-7 cells and (B) MDA-MB-231 cells transfected with miR-26a were detected via western blotting. miR, microRNA; SHH, sonic hedgehog signaling molecule; SMO, smoothened, frizzled class receptor; GLI1, GLI family zinc finger 1 .

difference in the luciferase activity of the MT 3'-UTR of FAM98A was detected (Fig. 5C). Furthermore, western blotting was performed to verify how miR-26a regulated FAM98A, and the results indicated that the expression level of the FAM98A protein was decreased in miR-26a-overexpressing cells (Fig. 5B).

miR-26a suppresses the Hh signaling pathway. As previously stated, miR-26a attenuated the malignant characteristics and enhanced the sensitivity of cancer cells to docetaxel. It was found that miR-26a targeted FAM98A and downregulated FAM98A expression. Previous studies have reported that the dysregulation of the Hh pathway may promote cancer cell survival and invasion, and is associated with drug resistance $(19,20)$. Thus, it was investigated whether miR-26a may exert its effects via aberrant activation of the Hh pathway. The western blot analysis demonstrated that the expression levels of SHH, SMO and GII1 were significantly decreased in miR-26a-overexpressing cells (Fig. 6A and B).

\section{Discussion}

The dysregulated expression of miRNAs is generally considered as a factor closely associated with oncogenesis, tumor development and the resistance to radiotherapy and chemotherapy in various types of cancer $(21,22)$. miRNA expression levels in malignant tumors may be increased or decreased compared with those in normal tissues (6). Moreover, the differential expression of miRNAs may play a key role in cancer by regulating the expression levels of the encoded oncogenes or tumor suppressor genes (23). One miRNA may regulate multiple target genes that are involved in the regulation of the biological processes of different carcinomas. Thus, miRNAs may be valuable as novel biological treatment targets $(24,25)$.

The present study demonstrated that miR-26a expression was downregulated in breast cancer tissues compared with that in corresponding non-cancerous breast tissues. These results were in accordance with previous research, which indicated that

miR-26a is a tumor suppressor in breast cancer (13). Previous studies have revealed that miR-26a exerted antitumor effects in other malignancies. For example, Li et al (26) revealed that the increase in miR-26a expression induced cell apoptosis and inhibited cell proliferation and invasion by downregulating the expression level of Wnt5a. It was also found that F-box protein 11 (FBXO11) was a downstream target regulator of miR-26a and was upregulated in hepatocellular carcinoma (HCC) cells. miR-26a overexpression exerts a suppressive effect on HCC cell proliferation, migration and invasion via the negative regulation of FBXO11 (27). Guo et al (28) reported that miR-26a was downregulated and SERBP1 was upregulated in prostate cancer, they suppressed prostate cancer cell proliferation and motility, and may represent novel prognostic biomarkers.

In the present research, in order to further assess whether miR-26a contributes to breast cancer cell proliferation and progression, miR-26a was overexpressed via transfection with miR-26a mimics. The overexpression of miR-26a markedly decreased the proliferation, clone formation ability, migration and invasion of cancer cells. Moreover, the results of the present study suggested that miR-26a overexpression may be a potential strategy for reversing docetaxel resistance in patients with breast cancer.

The present study also examined the mechanism through which miR-26a regulates its downstream targets. TargetScan analysis was used to identify the targeting association between miR-26a and FAM98A. As expected, the overexpression of miR-26a inhibited the luciferase activity of WT 3'-UTR of FAM98A, while no changes were found in the luciferase activity of MT 3'-UTR of FAM98A. The results indicated that FAM98A was a direct downstream target of miR-26a. The western blot analysis revealed that FAM98A protein expression was significantly downregulated in response to aberrant overexpression of miR-26a. Therefore, FAM98A was selected to be further studied as a novel target of miR-26a. The results suggested that miR-26a may act as a tumor suppressor by regulating the expression of FAM98A. However, little is currently known regarding FAM98A. FAM98A is a new substrate and is methylated by PRMT1, the expression level of which was shown to be associated with the malignancy of cancer cells (14). PRMTs, which are abnormally expressed in numerous malignancies, have been associated with the progression of various types of cancer (15).

The Hh signaling pathway is important for mammalian embryonic development, and its main components include SHH, desert hedgehog signaling molecule, Indian hedgehog signaling molecule, patched 1, Smo, GLI1 and GLI2 (29). Accumulating evidence has indicated that abnormal activation of the Hh pathway results in tumor cell survival, proliferation, stem cell maintenance and chemotherapy resistance (30). It has also been reported that multiple PRMTs (PRMT1, PRMT5 and PRMT7) regulate the activity of GLI, which is the downstream effector of the Hh pathway in cancer cells (31). Wang et al (32) observed that GLI1 methylation was mediated by PRMT1, and that the removal of GLI1 methylation significantly reduces GLI1-related carcinogenic functions and attenuates gemcitabine resistance in pancreatic cancer cells. The present study identified that miR-26a downregulated the expression level of FAM98A, and it also suppressed the expression of SHH, SMO and 
GLI1. Therefore, these results suggested that miR-26a may inhibit the malignancy of cancer cells and reverse the resistance to chemotherapy drugs via the depletion of FAM98A expression and the inactivation of the Hh signaling pathway. However, several questions remain unanswered. In the present study, it was confirmed that miR-26a targeted FAM98A mRNA and inhibited FAM98A protein expression; however, it remains unclear how FAM98A affects the proliferation, colony formation, migration and invasion of breast cancer cells. Then, it was further demonstrated that miR-26a suppressed the expression of SHH, SMO and GLI1, but it remains unknown whether miR-26a expression is associated with chemosensitivity. Therefore, further functional experiments should be conducted in the future to validate the present findings.

In conclusion, the expression miR-26a was found to be decreased in breast cancer cells and tissues in the present study, which in turn decreased cancer cell proliferation, clone formation ability, invasion and migration, and improved the sensitivity of cancer cells to docetaxel. It was also observed that miR-26 targeted FAM98A and downregulated its expression. Furthermore, it was demonstrated that miR-26 inhibited the Hh signaling pathway. Thus, it may be inferred that miR-26a downregulates the expression of FAM98A and inactivates the Hh signaling pathway to function as a tumor suppressor in breast cancer by inhibiting cell proliferation and cancer progression. However, further in-depth research is required in order to further elucidate the underlying mechanisms.

\section{Acknowledgements}

Not applicable.

\section{Funding}

The present study was supported by the New Medicine Foundation of University of Science and Technology of China (grant no. WK9110000058).

\section{Availability of data and materials}

The datasets generated and/or analyzed during the present study are available from the corresponding author on reasonable request.

\section{Authors' contributions}

TL and ZMW performed the experiments and analyzed the data. MHD helped with the collection of tissue specimens. JJW performed part of the RTqPCR and western blotting experiments. YYP contributed to the conception of the study and gave final approval of the version to be published. YYP and TL have verified the authenticity of the raw data. All the authors read and approved the final manuscript.

\section{Ethics approval and consent to participate}

This study was approved by the Ethics Committee of the First Affiliated Hospital of University of Science and Technology of China (approval no. 2019-ky086). All the patients provided written informed consent to participate in the study.

\section{Patient consent for publication}

Not applicable.

\section{Competing interests}

The authors declare that they have no competing interests.

\section{References}

1. Siegel RL, Miller KD and Jemal A: Cancer statistics, 2019. CA Cancer J Clin 69: 7-34, 2019.

2. Baltzer PAT, Kapetas P, Marino MA and Clauser P: New diagnostic tools for breast cancer. Memo 10: 175-180, 2017.

3. Gomez-Miragaya J, Moran S, Calleja-Cervantes ME, Collado-Sole A, Pare L, Gomez A, Serra V, Dobrolecki LE, Lewis MT, Diaz-Lagares A, et al: The altered transcriptome and DNA methylation profiles of docetaxel resistance in breast cancer PDX models. Mol Cancer Res 17: 2063-2076, 2019.

4. Huang P, Li F, Li L, You Y, Luo S, Dong Z, Gao Q, Wu S, Brunner $\mathrm{N}$ and Stenvang J: lncRNA profile study reveals the mRNAs and lncRNAs associated with docetaxel resistance in breast cancer cells. Sci Rep 8: 17970, 2018.

5. Fan L, Strasser-Weippl K, Li JJ, St LJ, Finkelstein DM, Yu KD, Chen WQ, Shao ZM and Goss PE: Breast cancer in China. Lancet Oncol 15: e279-e289, 2014.

6. Rupaimoole R and Slack FJ: MicroRNA therapeutics: Towards a new era for the management of cancer and other diseases. Nat Rev Drug Discov 16: 203-222, 2017.

7. Guo H, Ingolia NT, Weissman JS and Bartel DP: Mammalian microRNAs predominantly act to decrease target mRNA levels. Nature 466: 835-840, 2010.

8. Jeffries J, Zhou W, Hsu AY and Deng Q: miRNA-223 at the crossroads of inflammation and cancer. Cancer Lett 451: 136-141, 2019.

9. Si W, Shen J, Zheng H and Fan W: The role and mechanisms of action of microRNAs in cancer drug resistance. Clin Epigenetics 11: 25, 2019.

10. He XH, Zhu W, Yuan P, Jiang S, Li D, Zhang HW and Liu MF: miR-155 downregulates ErbB2 and suppresses ErbB2-induced malignant transformation of breast epithelial cells. Oncogene 35: 6015-6025, 2016.

11. Jafri MA, Al-Qahtani MH and Shay JW: Role of miRNAs in human cancer metastasis: Implications for therapeutic intervention. Semin Cancer Biol 44: 117-131, 2017.

12. Chen J, Zhang K, Xu Y, Gao Y, Li C, Wang R and Chen L: The role of microRNA-26a in human cancer progression and clinical application. Tumour Biol 37: 7095-7108, 2016.

13. Huang ZM, Ge HF, Yang CC, Cai Y, Chen Z, Tian WZ and Tao JL: MicroRNA-26a-5p inhibits breast cancer cell growth by suppressing RNF6 expression. Kaohsiung J Med Sci 35: 467-473, 2019.

14. Akter KA, Mansour MA, Hyodo T, Ito S, Hamaguchi M and Senga T: FAM98A is a novel substrate of PRMT1 required for tumor cell migration, invasion, and colony formation. Tumour Biol 37: 4531-4539, 2016.

15. Akter KA, Mansour MA, Hyodo T and Senga T: FAM98A associates with DDX1-C14orf166-FAM98B in a novel complex involved in colorectal cancer progression. Int J Biochem Cell Biol 84: 1-13, 2017.

16. Li Z, Li N, Sun X and Wang J: FAM98A promotes cancer progression in endometrial carcinoma. Mol Cell Biochem 459: 131-139, 2019.

17. Zheng R, Liu Q, Wang T, Wang L and Zhang Y: FAM98A promotes proliferation of non-small cell lung cancer cells via the P38-ATF2 signaling pathway. Cancer Manag Res 10: 2269-2278, 2018.

18. Livak KJ and Schmittgen TD: Analysis of relative gene expression data using real-time quantitative PCR and the 2(-Delta Delta C(T)) method. Methods 25: 402-408, 2001.

19. Katoh M: Genomic testing, tumor microenvironment and targeted therapy of Hedgehog-related human cancers. Clin Sci (Lond) 133: 953-970, 2019. 
20. Riaz SK, Khan JS, Shah STA, Wang F, Ye L, Jiang WG and Malik MFA: Involvement of hedgehog pathway in early onset, aggressive molecular subtypes and metastatic potential of breast cancer. Cell Commun Signal 16: 3, 2018.

21. Ma L: MicroRNA and Metastasis. Adv Cancer Res 132: 165-207, 2016.

22. Braicu C, Raduly L, Morar-Bolba G, Cojocneanu R, Jurj A, Pop LA, Pileczki V, Ciocan C, Moldovan A, Irimie A, et al: Aberrant miRNAs expressed in HER-2 negative breast cancers patient. J Exp Clin Cancer Res 37: 257, 2018.

23. Fridrichova I and Zmetakova I: MicroRNAs contribute to breast cancer invasiveness. Cells 8: 1361, 2019.

24. Biswas S: MicroRNAs as therapeutic agents: The future of the battle against cancer. Curr Top Med Chem 18: 2544-2554, 2018.

25. Yang C, Tabatabaei SN, Ruan X and Hardy P: The dual regulatory role of MiR-181a in breast cancer. Cell Physiol Biochem 44: 843-856, 2017.

26. Li Y, Wang P, Wu LL, Yan J, Pang XY and Liu SJ: miR-26a-5p inhibit gastric cancer cell proliferation and invasion through mediated Wnt5a. Onco Targets Ther 13: 2537-2550, 2020.

27. Ma Y, Deng F, Li P, Chen G, Tao Y and Wang H: The tumor suppressive miR-26a regulation of FBXO11 inhibits proliferation, migration and invasion of hepatocellular carcinoma cells Biomed Pharmacother 101: 648-655, 2018.
28. Guo K, Zheng S, Xu Y, Xu A, Chen B and Wen Y: Loss of miR-26a-5p promotes proliferation, migration, and invasion in prostate cancer through negatively regulating SERBP1. Tumour Biol 37: 12843-12854, 2016.

29. Wu F, Zhang Y, Sun B, McMahon AP and Wang Y: Hedgehog signaling: From basic biology to cancer therapy. Cell Chem Biol 24: 252-280, 2017.

30. Hanna A and Shevde LA: Hedgehog signaling: Modulation of cancer properies and tumor mircroenvironment. Mol Cancer 15: 24, 2016.

31. Abe Y and Tanaka N: Fine-Tuning of GLI activity through arginine methylation: Its mechanisms and function. Cells 9: 1973, 2020

32. Wang Y, Hsu JM, Kang Y, Wei Y, Lee PC, Chang SJ, Hsu YH, Hsu JL, Wang HL, Chang WC, et al: Oncogenic functions of gli1 in pancreatic adenocarcinoma are supported by its PRMT1-Mediated methylation. Cancer Res 76: 7049-7058, 2016.

This work is licensed under a Creative Commons Attribution-NonCommercial-NoDerivatives 4.0 International (CC BY-NC-ND 4.0) License. 